\title{
Editor's Note: Special Section on Computing and Combinatorics
}

The Algorithmica journal gratefully acknowledges the editorial work of the following scholars on this special section dedicated to computing and combinatorics, based upon work from COCOON 2013:

Ding-Zhu Du

The University of Texas at Dallas

Professor Guochuan Zhang

Zhejiang University

The three papers in this section include:

Facets for Art Gallery Problems, Sándor P. Fekete, Stephan Friedrichs, Alexander Kröller, Christiane Schmidt

On Randomized Fictitious Play for Approximating Saddle Points Over Convex Sets, Khaled Elbassioni, Kazuhisa Makino, Kurt Mehlhorn, Fahimeh Ramezani

Improved Approximation Algorithms for the Facility Location Problems with Linear/Submodular Penalties, Yu Li, Donglei Du, Naihua Xiu, Dachuan Xu 